\title{
An Agent-Based Simulation Framework For Cognitive Radio Studies
}

\author{
Jacek Dzikowski \\ Illinois Institute of Technology \\ $10 \mathrm{~W} \cdot 31^{\text {st }}$ St. \\ Chicago, IL 60616 \\ 1.312.567.5123 \\ dzikjac@iit.edu
}

\author{
Cynthia Hood \\ Illinois Institute of Technology \\ $10 \mathrm{~W} \cdot 31^{\text {st }} \mathrm{St}$. \\ Chicago, IL 60616 \\ 1.312.567.3918 \\ hood@iit.edu
}

\begin{abstract}
The constantly growing number of wireless devices and applications makes efficient spectrum utilization critical. Cognitive radio technology offers a solution by employing opportunistic and adaptive selection of transmission parameters and communication strategies. Due to the lack of general simulation tools available for analyzing cognitive radio networks, we propose an agent-based framework for cognitive radio studies. Unlike existing simulation tools, our design facilitates modeling of the physical environment along with the behavior of a network of cognitive radios. The inclusion of real-life spectrum occupancy data collected by the IIT Spectrum Observatory brings the modeled environment very close to reality. Additionally, because of its agent-based nature, our framework enables to study emergent and behavioral aspects of large and heterogeneous cognitive radio networks, an important factor that is being constantly neglected. Preliminary results show that presented solution is promising, but requires more development.
\end{abstract}

\section{Categories and Subject Descriptors}

B.8.2 [Performance and reliability]: Performance Analysis and Design Aid; C. 4 [Performance of systems]: Modeling techniques; I.6.5 [Simulation and modeling]: Model development --Modeling methodologies

\section{General Terms}

Management, Measurement, Documentation, Performance, Design, Experimentation.

\section{Keywords}

Cognitive radio, wireless network, agent based simulation and modeling, spectrum observatory, multi-agent system

\section{INTRODUCTION}

For years, exclusive band licensing has been a standard for

Permission to make digital or hard copies of all or part of this work for personal or classroom use is granted without fee provided that copies are not made or distributed for profit or commercial advantage and that copies bear this notice and the full citation on the first page. To copy otherwise, or republish, to post on servers or to redistribute to lists, requires prior specific permission and/or a fee.

SIMUTools 2009, Rome, Italy.

Copyright 2009 ICST, ISBN 978-963-9799-45-5. regulatory bodies such as the Federal Communications Commission (FCC). This fixed, time independent approach, however, was shown to be non-optimal, resulting in large portions of certain licensed frequency bands remaining underutilized most of the time [1]. Results obtained from spectrum observatory studies, including the one conducted at the Illinois Institute of Technology (IIT) [2], further support this statement. On the other hand, unlicensed and publicly available bands constitute a relatively small portion of the entire spectrum. Rapid growth in the number of wireless devices and applications in recent years makes this already limited resource even scarcer.

The FCC has tried to address the problem by proposing three fundamental directions (unlicensed spectrum, underlays and overlays [3][4]) towards spectrum utilization improvement. Out of these three, the concept of overlays (i.e. cognitive radio) is particularly promising, albeit challenging. Cognitive radio [5] was defined primarily as an extension of the software-defined radio notion [6] and envisioned as being able to make its own choices as to the network, modulation, and/or coding parameters based on its surroundings. Such radios could even make decisions based on the availability of nearby collaborative nodes, or on the regulations dictated by their current location and spectral conditions. This opportunistic and adaptive approach makes cognitive radio perfectly suited for improving spectrum utilization efficiency and flexibility. Clearly, cognitive radio technology offers an effective solution to that problem, yet it requires further development and study in several involved areas.

Establishment of concrete policies, regulations, and standards as well as devising effective algorithms requires observation of spectrum usage patterns and anomalies. Collecting and analyzing real life spectrum occupancy data through observatories [2] in several US urban areas is a major step towards greater understanding of related physical phenomena. Similarly, wireless interference studies deliver another important building block in cognitive radio know-how.

We are not aware of any simulation or software modeling framework that has been designed with cognitive radio studies in mind that considers spectrum occupancy data and wireless interference as well. Even though some of the techniques and assumptions with respect to wireless networks are criticized for their inadequacy [7], network simulation tools are commonly accepted as a reliable means of scientific evaluation. This is particularly true when the researched technology is still a few steps away from being widely deployed. Unfortunately, existing 
wireless network simulators offer little to no support for simulating the underlying physical environment. Ignoring this factor partially or completely affects the simulation results. In the case of cognitive radio, lack of spectrum occupancy models prohibits interested users from using them completely. Clearly, a new software framework would be a useful tool for researchers.

Another motivation behind the proposed simulation framework is the fact that cognitive radio networks are currently not researched from a behavioral standpoint. Cognitive radio networks will be comprised of a large number of heterogeneous elements that are expected to interact in an unpredictable and, most likely, chaotic fashion. Having some basic knowledge and expectations with respect to the entire system behavior under given circumstances, would certainly be helpful when designing dynamic spectrum allocation schemes and other related mechanisms. One of the goals of the proposed framework is exploiting the agent-based modeling features to acquire this knowledge.

Finally, the ability to use real-life spectrum occupancy data (as collected by spectrum observatories such as the one described in [2]) to model the environment in which cognitive radio networks operate makes this layer (i.e. environment) of the framework much more realistic. This approach allows for running wireless network models in a quasi-real world environment, without the necessity to design and develop expensive and time-consuming experiments with custom hardware.

The work presented here is an attempt to create a cohesive agentbased software framework for cognitive radio studies capable of including spectrum occupancy data. The following sections describe the reasoning behind selecting agent-based paradigm (section 2), present related work in the field (section 3), provide a detailed description of the software model/framework (section 4) present experimental results (section 5) and finally discuss obtained results and future work (sections 6 and 7, respectively).

\section{WHY AN AGENT-BASED APPROACH?}

A multi agent based model (MABM) is a computational model for simulating the mutual interactions of a group of autonomous entities (agents) constituting a system, providing a perspective to study their effects on the entire system that they are a part of. As such, the MABM has proven to be particularly effective in representing complex, dynamic systems, otherwise difficult or even impossible to simulate using other techniques. The agent-based methods have been particularly successful in studying complex systems exhibiting emergent behavior, i.e. a behavior arising from a multiplicity of fairly simple interactions.

The agent-based approaches are, however, neither new nor only within the domain of computer network modeling and simulation. As a matter of fact, other methods, such as equation-based, discrete event or object-oriented simulation are considered successful and prevalent in a majority of available wireless network models/simulators. Furthermore, hybrid solutions such as extending discrete event simulation with agent-based model(s) are possible [8], although they are cumbersome.

The decision as to which method should be applied in developing a software framework for cognitive radio studies is based on the nature of the cognitive radio network. Let us take a look at such a network as a system. It usually consists of a large number of homogeneous or heterogeneous, autonomous, decentralized entities (cognitive radios). It also follows the definition of the multi-agent system as given by Jennings et al. [9] and having the following characteristics:

- each agent has a limited viewpoint (incomplete information, or capabilities for solving a problem),

○ there is no global system control and data is decentralized,

○ computation is asynchronous.

Finally, the idea of cognitive radio as originally presented by Mitola [5], considered "smart" radio to be an agent capable of intelligent channel selection If MABM methodology is used, it is easy to observe that both the model and the physical system have the same or very similar structures.

This choice remains justified, when possible modeling strategies are compared with the target application in mind. Parunak et al. [10] compared equation-based and agent-based approaches and determined that "....agent-based modeling is most appropriate for domains characterized by a high degree of localization and distribution and dominated by discrete decision. Equation-based modeling is most naturally applied to systems that can be modeled centrally, and in which the dynamics are dominated by physical laws rather than information processing." The work of Davidsson [11] extended the comparison with object-oriented and discrete event simulation, concluding the first to be a limited version of MABM and the latter to be inferior for the discussed type of models.

The benefits of using MABM are the following:

○ It supports structure preserving modeling and implementation of the simulated reality,

- It supports modeling of pro-active behavior,

- It supports distributed computation in a very natural way. Since each agent is typically implemented as a separate piece of software corresponding to a process (or a thread), it is straightforward to let different agents run on different machines. This allows for better performance and scalability.

- Since each agent typically is implemented as a separate process and is able to communicate with any other agent using a common language, it is possible to add or remove agents during a simulation without interruption. This enables extremely dynamic simulation scenarios.

As far as weaknesses are concerned, multi agent-based models (MABMs) tend to be more resource and computational time intensive. MABMs are less appropriate for event driven simulations, where some form of centralized coordination and a large amount of synchronization between agents is required [11]. MABMs are also known to be fairly difficult to verify and validate.

\section{RELATED WORK}

While cognitive radio is no longer purely a theoretical concept, it is still far from successful mass deployment. In fact, aside from military applications [12], it has not as yet left research labs as a complete technology. 
Nevertheless, improving wireless spectrum utilization by means of cognitive radio as a research problem has attracted significant attention over the years. First attempt at standardization have already been made. The IEEE 802.22 working group [13] is developing a standard for networks operating in unused television channels. Another group focuses on $\mathrm{WiFi}$ and Bluetooth coexistence in unlicensed bands [14]. Also, a significant body of experimental and simulation work in the field has been accumulated, both in software and hardware domains with emphasis on the following issues:

- Selecting radio configuration parameters: power, frequency, encoding scheme, packet format etc. that will fulfill communication needs depending on the current environment conditions. (with application of game theory [15], genetic algorithms [16], machine learning, Petri Nets [17],

- Detecting other users (emphasis on primary users) [18],

- Fair resource sharing promotion. [19].

Interestingly, in spite of the close relationship to wireless network development and regardless of the direction and sophistication level, the cognitive radio experiments do not involve any existing wireless network simulation packages. Although it may seem strange at first, this fact is not a surprise at all once we take a closer look at the wireless network modeling domain.

Despite the aforementioned controversy surrounding wireless network simulation software credibility, it is still a standard research approach in this area. Existing non-agent-based simulation packages fall into two categories:

- general network simulators with additional wireless networking components (OPNET [20], NS-2 [21], OMNET++ [22]) or

○ wireless network specific (J-Sim [23], SENS [24], ATEMU [25]).

The problem is that available wireless network simulators and models frequently neglect (completely or to some extent) the physical environment. Only some of them include provide limited means of simulating the physical environment and its impact upon wireless network in two forms:

○ wireless channel interference models (NS-2, OPNET),

- various signal propagation/path loss models (J-Sim, SENS, ATEMU).

These simulators do not provide explicit mechanisms for incorporating spectrum occupancy data into the experiment. Similarly, the actual impact of the wireless device on its environment is not considered. Therefore, constructing cognitive radio-related models and experiments within these frameworks is most likely impractical, considering the lack of proper infrastructure.

Dedicated agent-based wireless network models (a much more natural choice for this particular application, as it was previously explained) only begin to appear in development stage, such as WISDOM [26]. This particular framework, however, focuses on wireless sensor network studies. Typically models used by researchers in cognitive radio studies are custom made for a given problem. This makes comparing and expanding concepts cumbersome without a single cohesive simulation framework.
Fortunately, such a framework does not have to be developed from scratch. There exist several agent-based modeling toolkits such as NetLogo [27], Repast [28], and AnyLogic [29], to name the most popular and general ones. Their interaction-oriented approach makes them flexible enough to construct models for a variety of problems from many fields, including cognitive radio.

Finally, historical real-life spectrum occupancy data is already available [2] to be embedded in to the framework. Although, at this point data comes from a single location (IIT Tower), a mobile spectrum observatory (i.e. spectrum observatory setup installed on a truck) is being implemented to collect spectrum occupancy information from multiple locations in Chicago. In addition, simple interference source models exist [30].

\section{BUILDING THE FRAMEWORK}

The proposed agent-based framework for cognitive radio study was designed by following the methodology outlined in [31] (see fig.1). A simplified view of the model is presented in fig.2.

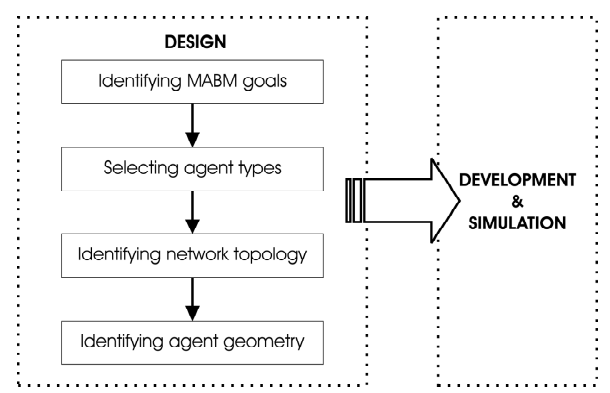

Figure 1. Multi-agent based model design, development and application methodology outline (based on: [31]).

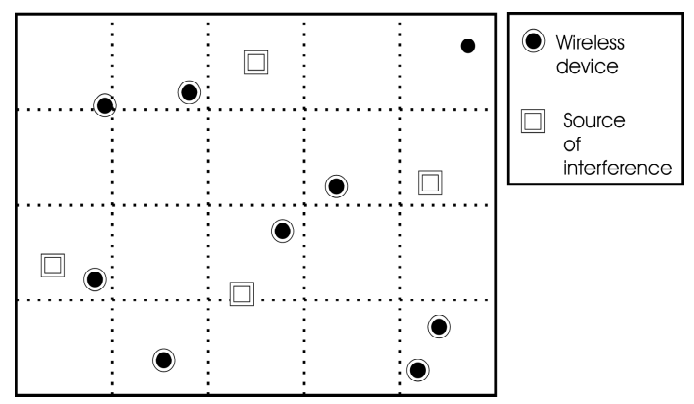

Figure 2. Simplified model view.

\subsection{Multi-Agent System Goals}

The proposed framework was designed with the following research goals in mind:

- Enhancing existing wireless network modeling and simulation strategies with environment data,

- Observing and analyzing the emergent behavior of the cognitive radio network for strategy building purposes,

- Ability to embed real spectrum occupancy data history within the model framework,

○ Testing a variety of channel selection mechanisms,

- Studying the impact of factors such as number of devices, transmission power and frequency, and interference on spectrum occupancy, 


\subsection{Selecting Agent Types}

The entire framework is built around three types of agents that define the environment as well as cognitive radio network(s):

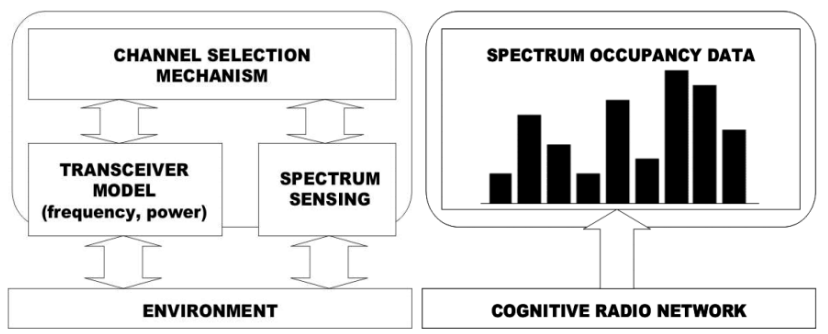

Figure 3. Cognitive radio and environment agents.

○ Cognitive radio agent: decision-making (intelligent) agent (schematics - see fig. 3), which interacts with the environment and other radio agents through a spectrum sensing mechanism (sensors: spectrum data obtained directly from corresponding environment agent) and transceiver (actuator: using selected frequency and power). Decision making is performed by a programmable channel selection mechanism (agent program) which can be rulebased for a simple reflex agent up to sophisticated with adaptive and learning capabilities. The cognitive radio agent is placed in the environment by means of its location parameter (Cartesian coordinates),

○ Environment agent (see fig.3): simple, non-intelligent (i.e. without any decision-making capabilities) agent interacting with cognitive radio agents through spectrum occupancy data (cognitive radio agents "sense" location specific information and affect it by its transmission). Spectrum occupancy data is defined as a table of band/occupancy pairs. Spectrum data can be loaded from the spectrum observatory datafile. An environment agent corresponds to a square part of the rectangular grid, defined by four Cartesian coordinates,

- Interference source agent: simple, non-intelligent agent modeled after microwave oven model described in [30].

\subsection{Identifying Network Topologies}

All agents operating within the framework are arranged in one of the following topologies, depending on their purpose:

- Cognitive radio network topology: The cognitive radio network considered in the model consists of a set of $N$ fixed transmitting-receiving cognitive radio agents, uniformly distributed in a rectangular environment (dimensions: $D x \mathrm{x} D y$ ). Fig. 2 shows an example of a network realization. The nodes measure the spectrum availability and decide on the transmission channel. It is assumed that there are $K$ available frequency channels, with $K<N$,

○ Environment topology: Environment agents are arranged in a rectangular grid as shown on fig. 2.

\subsection{Identifying Agent Topologies}

Agent geometry, i.e. geometry of agent interactions, within the considered framework, is defined with two different contexts in mind:
- Cognitive radio network context (see fig. 4): the geometry of actual network communication between cognitive radio agents/nodes.

- Environment context (see fig. 4): the geometry of the cognitive radio agent(s) and its corresponding environment agent interactions. Interactions are established by means of location data assigned to cognitive radio agents, i.e. given cognitive radio agent $C$ physical location by $(x, y)$ and environment agent $E$ physical boundaries by $(X 1, Y 1, X 2$, $Y 2), C$ will interact with $E$ if $X 1<x \leq X 2$ and $Y 1<y \leq Y 2$.

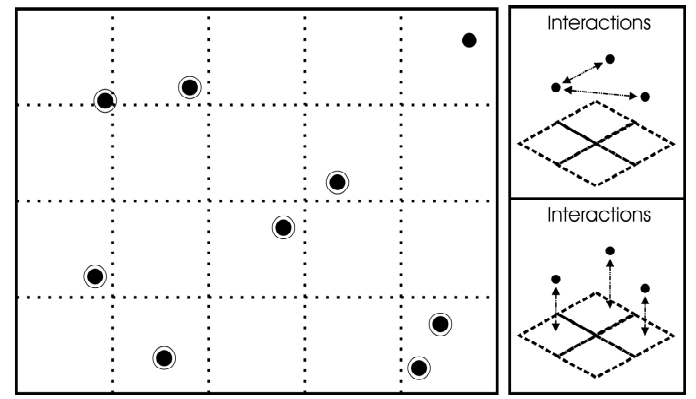

Figure 4. Agent topologies: cognitive radio network context (top), environment context (bottom).

\subsection{Modeling Toolkit}

The entire framework was programmed and developed using Repast Simphony [28], which is a free and open source agentbased modeling toolkit that simplifies model creation and use. This choice was dictated by previous experience with the tool, as well as expected testing of MADCABS multi-agent control system [32] that was developed using the same tool.

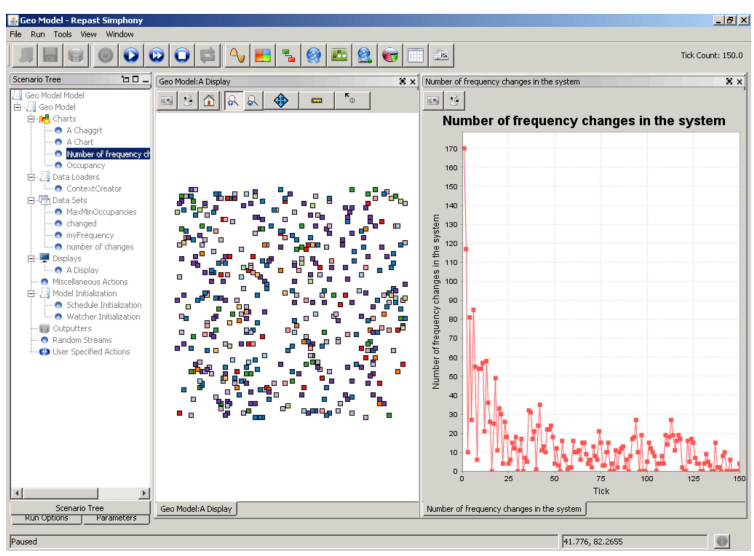

Figure 5. Software framework screenshot.

\section{PRELIMINARY RESULTS}

\subsection{Framework Verification}

In order to verify operation of fundamental framework mechanisms (agent creation/operation, topologies, interactions) a following test was constructed. Given a $2 \times 1$ environment grid with $K=2$ initially unoccupied channels and two networks (i.e. groups of devices transmitting at the same frequency; with $N=10$ devices each) uniformly distributed in the environment:

$\circ$ at time $t=10$ network 1 devices enter the environment, 
a)

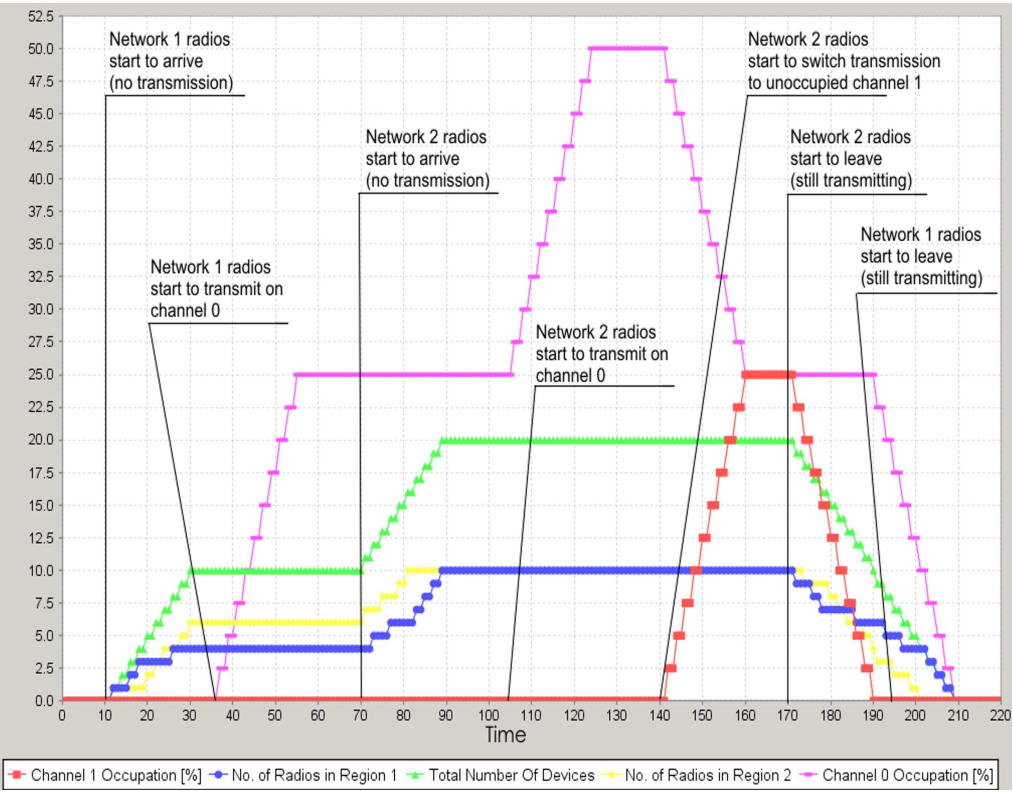

b)

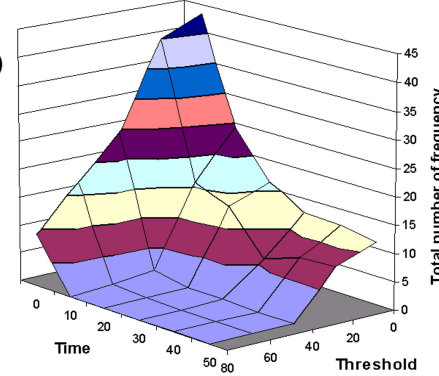

c)

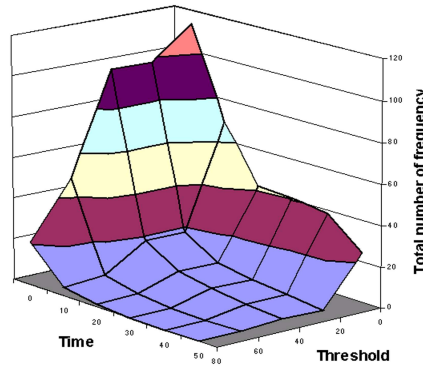

Figure 6. Preliminary results: a) verification outcome, $b, c)$ scenario results $(b: N=50, K=10 ; c: ~ N=125, K=10)$

$\circ$ at time $t=35$, network 1 devices start transmission (ch.0),

$\circ$ at time $t=70$ network 2 devices enter the environment,

$\circ$ at time $t=105$ network 2 devices start transmission (ch.0),

$\circ$ at time $t=140$ network 2 devices switch channel from 0 to 1 ,

0 at time $t=170(t=195)$ network $2(1)$ devices start to leave.

For simplicity, it is assumed that all devices transmit at the same power and each contributes $2.5 \%$ of total spectrum occupancy level. The outcome of this experiment is presented on fig. 6a. It depicts the effects of dynamically changing environment and agent networks. Also, it shows how changes in transmission parameters properly affect mutual interactions between agents.

\subsection{Simple Scenario Results}

Due to lack of available real-life experiment data, our framework cannot be completely validated at this time. However, a simple experiment was devised in attempt to partially validate the model and obtain preliminary results for a simplistic, "greedy" (i.e. each radio agent selects lowest occupied frequency), dynamic channel selection algorithm outlined below:

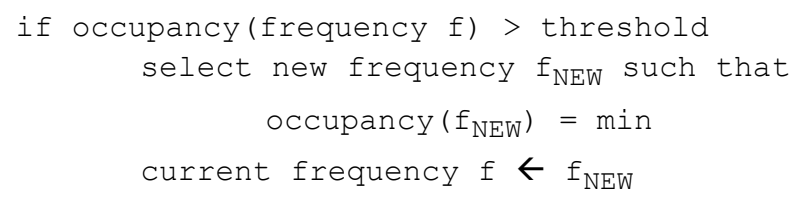

end

The environment is a $5 \times 5$ grid. Each part of the grid has $K$ available frequencies with a randomly assigned occupancy level ranging from 1 to 10 (100\% occupation). Cognitive radio network consists of $N$ homogeneous devices uniformly distributed over the environment. All devices constantly transmit with the same power and use the same threshold value. Transmission channel is randomly assigned to a device at the beginning. Channel selection is performed independently (i.e. without any negotiation).
The obtained results are presented in fig. 6b,c. Not surprisingly, increasing frequency selection threshold decreases the number of frequency changes. As intuitively expected, selecting low threshold reduces the number of remaining frequencies to choose from. Similarly, larger number of devices in the system increases the total number of frequency changes within the network. Also, an emergent behavior of the network can be observed. Simple low-level decision-making without communication between devices brings the entire network to a steady state, while none of them has this task as its goal.

\section{SUMMARY}

The presented work is an attempt to satisfy the existing demand for a flexible cognitive radio network simulation tool that can be used to address many currently investigated problems in this domain. On top of being a wireless network simulation framework, its underlying agent-based approach adds another dimension to related studies: it allows analyzing the behavioral aspects of the network. This particular element, and its importance with respect to large and dynamic systems (such as cognitive radio networks), is critical for analysis, development and successful deployment. Also, our framework is being designed to couple cognitive radio simulation with real-life spectrum occupancy data. This addition allows creating quasi-real environments for cognitive radio simulation. Altogether, described features bring more value to experimental work conducted using our tool and according to our knowledge this is most likely first simulation framework that includes those.

\section{FUTURE WORK}

Our main focus is on further development of the framework and refining details. Simultaneously, our efforts are directed towards finding reliable means of validating the framework. Also, we plan further integration with the spectrum observatory project and testing various known dynamic channel allocation methods described in literature. 


\section{REFERENCES}

[1] Federal Communications Commission Spectrum Policy Task Force, "FCC report of the spectrum efficiency working group," Tech. Rep., Federal Communications Commission, Washington, DC, USA, November 2002.

[2] Roberson, D.A., Hood, C., LoCicero, J.L., MacDonald, J.T., "Spectral Occupancy and Interference Studies in support of Cognitive Radio Technology Deployment," Networking Technologies for Software Defined Radio Networks, 2006, SDR '06, 1st IEEE Workshop on, Sept. 2006.

[3] Roberson, D.A., "Structural Support for Cognitive Radio System Deployment," in Proceedings of Cognitive Radio Oriented Wireless Networks and Communications, 2007.

[4] "Facilitating Opportunities for Flexible, Efficient, and Reliable Spectrum Use Employing Cognitive Radio Technologies" FCC Report and Order, FCC-05-57A1, March $11,2005$.

[5] Mitola, J., "Cognitive Radio: An Integrated Agent Architecture for Software Defined Radio," Dissertation, Royal Institute of Technology (KTH), May 8, 2000.

[6] Mitola, J., "Software Radio Architecture," John Wiley \& Sons, 2000.

[7] Kotz, D., Newport, C., Gray, R.S., Liu, J., Yuan, Y., Elliot, C., "Experimental Evaluation of Wireless Simulation Assumptions," in Proc. of MSWim'04, pp. 78-82, Oct. 2004.

[8] Dubiel, B.; Tsimhoni, O., "Integrating agent based modeling into a discrete event simulation," Simulation Conference, 2005 Proceedings of the Winter, vol., no., pp. 9 pp.-, 4-7 Dec. 2005

[9] Jennings, N., Sycara, K., Wooldridge, M., "A roadmap of agent research and development," in Journal of Autonomous Agents and Multi-Agent Systems, Volume 1, Number 1, March, 1998 pp. 275-306.

[10] Parunak, H.V.D., Savit, R., Riolo, R.L., “Agent-Based Modeling vs. Equation-Based Modeling: A Case Study and Users' Guide," in J.S. Sichman, R. Conte, and N. Gilbert. (Eds.), Multi-Agent Systems and Agent-Based Simulation, Springer Verlag, 1998.

[11] Davidsson, P., "Multi Agent Based Simulation: Beyond social simulation," In Moss, S. and Davidsson, P. (Eds.) Multi Agent Based Simulation, Springer Verlag LNCS series, Vol. 1979.

[12] McHenry, M.; Livsics, E.; Thao Nguyen; Majumdar, N., "XG Dynamic Spectrum Sharing Field Test Results," New Frontiers in Dynamic Spectrum Access Networks, 2007. DySPAN 2007. 2nd IEEE International Symposium on , vol., no., pp.676-684, 17-20 April 2007

[13] http://www.ieee802.org/22/

[14] http://www.ieee802.org/15/

[15] Saraydar, C. U., Mandayam, N. B., and Goodman, D. J., "Efficient power control via pricing in wireless data networks", IEEE Transactions on Communications, vol. 50, pp. 291-303, February 2002

[16] Rondeau, T. W., Le, B., Rieser, C. J., Bostian, C. W., "Cognitive radios with genetic algorithms: Intelligent control of software defined radios," in SDR Forum Technical Conference, 2004.

[17] Viessmann, A. et al "Petri Net Based Controller Concept For Cognitive Radios in Wireless Access Networks" in Journal of Communications, Vol. 2, No. 2, March 2007.

[18] Fehske, A.; Gaeddert, J.; Reed, J.H., "A new approach to signal classification using spectral correlation and neural networks," New Frontiers in Dynamic Spectrum Access Networks, 2005. DySPAN 2005. 2005 First IEEE International Symposium on , vol., no., pp.144-150, 8-11 Nov. 2005.

[19] Queseth, O., Coexistence and Competition in Unlicensed Spectrum. PhD thesis, KTH Stockholm, 2005.

[20] http://www.opnet.com

[21] http://www.isi.edu/nsnam/ns/

[22] Varga, A., "Using the OMNeT++ discrete event simulation system in education," Education, IEEE Transactions on , vol.42, no.4, pp.11 pp.-, Nov 1999.

[23] Ahmed Sobeih; Wei-Peng Chen; Hou, J.C.; Lu-Chuan Kung; Ning Li; Hyuk Lim; Hung-Ying Tyan; Honghai Zhang, "JSim: a simulation environment for wireless sensor networks," Simulation Symposium, 2005. Proceedings. 38th Annual, vol., no., pp. 175-187, 4-6 April 2005

[24] Sundresh, S.; Wooyoung Kim; Agha, G., "SENS: a sensor, environment and network simulator," Simulation Symposium, 2004. Proceedings. 37th Annual, vol., no., pp. 221-228, 18-22 April 2004.

[25] Polley, J.; Blazakis, D.; McGee, J.; Rusk, D.; Baras, J.S., "ATEMU: a fine-grained sensor network simulator," Sensor and Ad Hoc Communications and Networks, 2004. IEEE SECON 2004. 2004 First Annual IEEE Communications Society Conference on , vol., no., pp. 145-152, 4-7 Oct. 2004.

[26] Hock Beng Lim, Bang Wang, Cheng Fu, Phull, A.; Di Ma, "WISDOM: Simulation Framework for Middleware Services in Wireless Sensor Networks," Consumer Communications and Networking Conference, 2008. CCNC 2008. 5th IEEE, vol., no., pp.1269-1270, 10-12 Jan. 2008

[27] http://ccl.northwestern.edu/netlogo/

[28] http://repast.sourceforge.net/

[29] http://www.xjtek.com/

[30] Taher, T. M., Al-Banna, A. Z., LoCicero, J. L., Ucci, D. R., "Characteristics of an Unintentional Wi-Fi Interference Device - The Residential Microwave Oven," in Proceedings of IEEE Military Communications Conference, Oct. 2006.

[31] Kilicay, N.H.; Dagli, C.H., "Emergence and artificial life," Engineering Management Conference, 2003. IEMC '03. Managing Technologically Driven Organizations: The Human Side of Innovation and Change, vol., no., pp. 580584, 2-4 Nov. 2003.

[32] Tetiker, M.D., Artel, A., Tatara, E., Teymour, F., North, M., Hood, C., Cinar, A., "Agent-based system for reconfiguration of distributed chemical reactor network operation," American Control Conference, 2006 , vol., no., pp. 6 pp.-, 14-16 June 2006. 